\title{
Analytical wall function including roughness corrections
}

\author{
F. Chedevergne \\ ONERA, The French Aerospace Lab, F-31055 Toulouse, France
}

\begin{abstract}
Inspired by the work of Aupoix $[1,2]$ and relying on the analytical wall function of Suga et al. [3], this paper proposes a modified version of the wall model capable of accounting for roughness effects. The thermal correction was enhanced to capture roughness effects due to the increase of the wetted surface of the walls. A derivation of the model adapted to configurations with very large roughness is also proposed. The new model is compared to the former analytical wall function formulation using several rough configurations for which experimental data are available. The validation test cases have been chosen to highlight the improvements brought by the present work.
\end{abstract}

Keywords:

Analytical wall function, Heat transfer, Turbulence modelling, Wall roughness

\section{Introduction}

Investigations on effects of roughness on boundary layers date back to the early 1930's with major contributions by Nikuradse [4, 5]. Roughness is known to increase wall friction and heat transfer. In the full rough regime,

Email address: francois.chedevergne@onera.fr (F. Chedevergne) 
the surface friction increase is related to the pressure drag induced by the presence of rough elements on walls. As for heat transfer, combined effects of increased near wall turbulence levels and increased wetted surface are responsible of the rise. Owing to the distinct types of mechanisms involved in the friction and heat transfer increases, wall roughness cause less heat transfer growth than friction growth rendering the Reynolds analogy no longer valid on rough walls.

Even though a large and increasing number of industrial applications (wear of the blades in turbines, icing accretion on wings, ablation on reentry vehicles, etc.) require to account for roughness effects, most of the efforts in the fluid mechanics community concentrate on modeling boundary layers over smooth surfaces. In recent years, progress has been made in the development of turbulence models capable of reproducing roughness effects and applicable in an industrial context. Among methods used to model rough flows, the equivalent sand grain method revealed to be the most appropriate to engineering applications. The method is made of two steps. First, using correlations the rough surface is reduced to an equivalent surface covered by sand grains of height $h_{s}$ that will produce the same friction increase. Then, an ad-hoc correction driven by $h_{s}$ is made on a turbulence model in order to artificially enhance turbulence in the wall region and reproduce the friction increase. On this basis, Aupoix [1] built a correction for the SST $k-\omega$ turbulence model of Menter [6]. This first correction has been completed with an additional correction [2] to account for thermal effects due to roughness, since the Reynolds analogy cannot be used. 
Combined, both corrections work remarkably on a variety of heated boundary layers over rough walls. But such an approach requires the use of fine meshes at walls inasmuch as it is a low-Reynolds number (LRN) approach. For some industrial applications, there is a crucial need to reduce CPU costs while making use of coarse meshes. For instance, in the framework of the development of ONERA's icing suites IGLOO2D [7, 8] and IGLOO3D [9], which involve a large number of cycles of a flow solver, different solutions are currently being studied to avoid having resource to the use of wall-refined meshes. The first option consists in coupling a boundary layer code $[10,11]$ to an Euler solver; the second being the development of a wall function approach in a Navier-Stokes environment.

Starting from the analytical wall function (AWF) developed by Craft et al. [12] which permits the computation of wall flows while including many physical effects (buoyancy, laminarization, properties temperature dependency), Suga et al. [3] introduced modifications to account for rough walls. A simplified version of the original AWF by Craft et al. was used in Suga's work which do not include all refinements. Although very satisfactory results were obtained by Suga et al. some points deserve further improvements. More specifically, Suga's model has been validated on applications where roughness effects where limited, i.e. for which $h_{s}$ values were not too large. In addition, wetted effects that are known to play a role in the heat transfer over rough walls are not accounted for in Suga's model. Therefore, the present study investigates the possibility of matching the work by Suga et al. with 
the corrections initiated by Aupoix to extend the validity range of the AWF. First a reminder of the principles driving Aupoix's corrections is made. Subsequently, the AWF formulation of Suga et al. is recapped. Finally, a new proposal is formulated and validation cases highlighting the strengths of the present AWF are performed.

\section{Aupoix's roughness corrections}

Only distributed roughness will be considered, i.e. where characteristic lengths (height, span and spacing) are small compared to boundary layer thickness. Additionally, only $k$-type roughness [13], for which effects are related to their heights, will be treated in the following study. Relying on the classical description of turbulent boundary layer over rough wall initiated by Nikuradse [4, 5] and previous works [14, 15], Aupoix developed a dynamic correction [1] recreating the friction increase due to roughness in the framework of the $k-\omega$ SST model [6]. Then, since the Reynolds analogy no longer holds for turbulent boundary layer over rough walls, Aupoix enhanced his work with a thermal correction [2]. Both aspects are briefly reminded hereinafter.

\subsection{Dynamic correction}

Nikuradse $[4,5]$ pointed out that above roughness the logarithmic law is preserved but shifted. The velocity profile in wall variables $u^{+}$reads:

$$
u^{+}=\frac{1}{\kappa} \ln y^{+}+C-\Delta u^{+} \quad u^{+}=\frac{u}{u_{\tau}}, \quad y^{+}=\frac{y u_{\tau}}{\nu}
$$


where $C=5.5$ and $\kappa$ is the Kármán constant. Note that Nikuradse $[4,5]$ retained $\kappa=0.4$ in his studies. However, in the present study the classical value $\kappa=0.41$ is used.

The friction velocity used to defined wall quantities in all the following is $u_{\tau}=\sqrt{\frac{\tau_{w}}{\rho}}, \tau_{w}$ being the wall shear stress. In Nikuradse's experiments, $\tau_{w}$ is determined from the measured pressure drop in a pipe. The shift $\Delta u^{+}$is related to the equivalent sand grain height $h_{s}^{+}=\frac{h_{s} u_{\tau}}{\nu}$. Multiple expressions have been derived in the literature and in the present work the compact form provided by Grigson [16] and obtained from Colebrook's data [17, 18] is retained:

$$
\Delta u^{+}=\frac{1}{\kappa} \ln \left(1+\frac{h_{s}^{+}}{\exp (3.25 \kappa)}\right)
$$

Starting from this fundamental observation on boundary layer profiles, Aupoix and Spalart [14] proposed a general strategy to reproduce the shift in turbulence models while artificially increasing turbulent viscosity $\mu_{t}$ at the wall. The leading principles are the following: first, a wall shift $y_{0}$ is introduced so that velocity gradients over rough and smooth surfaces satisfy:

$$
\left.\frac{\partial u_{r}^{+}}{\partial y^{+}}\right|_{y^{+}}=\left.\frac{\partial u_{s}^{+}}{\partial y^{+}}\right|_{y^{+}+y_{0}^{+}}
$$

where subscripts $r$ and $s$ refer to rough and smooth surfaces respectively. After integration along the wall normal it yields:

$$
u_{r}^{+}\left(y^{+}\right)=u_{s}^{+}\left(y^{+}+y_{0}^{+}\right)-u_{s}^{+}\left(y_{0}^{+}\right)
$$

The shift $\Delta u^{+}$is thus directly given by: 


$$
\Delta u^{+}=u_{s}^{+}\left(y_{0}^{+}\right)
$$

In turbulent boundary layers, considering a constant total shear, the momentum equation reduces to:

$$
\left(1+\mu_{t}^{+}\right) \frac{\partial u^{+}}{\partial y^{+}}=1
$$

with $\mu_{t}^{+}=\frac{\mu_{t}}{\mu}$.

Finally, from eq. (3) it becomes:

$$
\mu_{t_{r}}^{+}\left(y^{+}\right)=\mu_{t_{s}}^{+}\left(y^{+}+y_{0}^{+}\right)
$$

The initial search for the $\Delta u^{+}$shift has been transferred to the one for $y_{0}^{+}$that yields the desired eddy viscosity increase. In particular at the wall, $\mu_{t_{r_{w}}}$ may not be zero:

$$
\mu_{t_{r_{w}}}^{+}=\mu_{t_{s}}^{+}\left(y_{0}^{+}\right)
$$

In practice, once a relationship between $\Delta u^{+}$and $h_{s}^{+}$such as in eq. (2) is known, a smooth profile expression can be used to find the $y_{0}^{+}$that satisfies eq. (5). Then, considering the $k-\omega$ SST turbulence model, it is straightforward to get the $k_{s}^{+}\left(y_{0}^{+}\right)$and $\omega_{s}^{+}\left(y_{0}^{+}\right)$that yield the $k_{w}^{+}$and $\omega_{w}^{+}$values to be imposed at the wall to recover eq. (8). Turbulent scalars are made dimensionless with the friction velocity $u_{\tau}$ and the viscosity $\nu$ such that $k^{+}=\frac{k}{u_{\tau}^{2}}$ and $\omega^{+}=\frac{\omega \nu}{u_{\tau}^{2}}$. Expressions $k_{w}^{+}\left(h_{s}^{+}\right)$and $\omega_{w}^{+}\left(h_{s}^{+}\right)$have been obtained by Aupoix [1] and read: 


$$
\begin{aligned}
& k_{w}^{+}=\max \left(0 ; k_{0}^{+}\right) \\
& k_{0}^{+}=\frac{1}{\sqrt{\beta^{*}}} \tanh \left[\left(\frac{\ln \frac{h_{s}^{+}}{30}}{\ln 10}+1-\tanh \frac{h_{s}^{+}}{125}\right) \tanh \left(\frac{h_{s}^{+}}{125}\right)\right] \\
& \omega_{w}^{+}=\frac{300}{h_{s}^{+2}}\left(\tanh \frac{15}{4 h_{s}}\right)^{-1}+\frac{191}{h_{s}^{+}}\left[1-\exp \left(-\frac{h_{s}^{+}}{250}\right)\right]
\end{aligned}
$$

\subsection{Thermal correction}

The dynamic correction is an ad-hoc correction that reproduces the pressure effect on drag through an increase of the eddy viscosity at the wall. If no further correction is introduced in the $k-\omega$ SST turbulence model, the eddy thermal conductivity at the wall $\lambda_{t_{r_{w}}}$ is overestimated since the Reynolds analogy is no longer valid for rough surfaces. Using the dynamic correction (9) associated with a constant turbulent Prandtl number $P r_{t}$ this approach was proven to overestimate heat transfers [2]. A simple way to derive a thermal correction is to modify the turbulent Prandtl number by writting:

$$
P r_{t}=P r_{t_{\infty}}+\Delta P r_{t}
$$

where $\mathrm{Pr}_{t_{\infty}}$ is the standard turbulent Prandtl number value 0.9. Several parameters rule the correction developed by Aupoix [2], each one representing different physical behaviors. The thermal correction $\Delta P r_{t}$ must be restricted to a certain extent from the wall, i.e. within the roughness sublayer. The latter corresponds to the near wall region where the flow is strongly affected by the presence of roughness. Above the roughness sublayer, the flow is locally homogeneous in planes parallel to the wall. The roughness sublayer 
generally expands up to three to five times the mean roughness height $h$. An exponential decay involving this mean roughness height $h$ has been introduced into $\Delta P r_{t}$ to limit the extent of the correction. The equivalent sand grain height $h_{s}$, which provides the velocity shift $\Delta u^{+}$, allows to account for the turbulence diffusion, whereas wetted surface effects are accounted for using an additional parameter $S_{\text {corr }}$. The latter is the corrected wetted surface ratio defined using the surface geometry where troughs below the reference (melt-down surface) are neglected [2]. The final correction of Aupoix reads as follow:

$$
\begin{gathered}
\Delta P r_{t}=\mathcal{F}^{A} \exp (-y / h) \\
\mathcal{F}^{A}=A \Delta u^{+^{2}}+B \Delta u^{+} \\
A=\left(0.0155-0.0035 S_{\text {corr }}\right)\left[1-\exp \left(-12\left(S_{\text {corr }}-1\right)\right)\right] \\
B=-0.08+0.25 \exp \left(-10\left(S_{\text {corr }}-1\right)\right) \\
\Delta u^{+}=\Delta u^{+}\left(h_{s}^{+}\right)
\end{gathered}
$$

In the studied subsonic heated boundary layers configurations, $\Delta P r_{t}$ lies between 0 and 2 as shown on figure 3 .

Both dynamic and thermal corrections have been extensively validated $[1,2]$ in a boundary layer context using ONERA's code CLICET.

\section{Analytical wall function model}

Following the ideas of Craft et al. [12], Suga et al. [3] developed an extension of the AWF for turbulent flows over rough walls. For the sake of clarity, in the following the work by Craft et al. is refered as the original AWF. The 
main idea of the original AWF is to impose a linear evolution to the eddy viscosity in the internal region of the boundary layer. One of the advantage of this approach is that the logarithmic behavior is not imposed to the velocity profile. Nevertheless, in a zero pressure gradient (ZPG) boundary layer the expected logarithmic behavior will be recovered, due to the linearity of $\mu_{t}$. The model reads:

$$
\mu_{t}=\rho C_{\mu}^{1 / 4} \sqrt{k} l=\rho C_{\mu}^{1 / 4} \sqrt{k} \kappa y \approx \alpha \mu y^{*}
$$

where $C_{\mu}=0.09$. Let's denote $\mathrm{P}$ the center of a wall cell. $y^{*}=\frac{y \sqrt{k_{P}}}{\nu}$ is a characteristic Reynolds number for wall cells. Eq. (12) originates from the fact that $k_{P}$ can be used to define a turbulent velocity scale that yields $u_{\tau} \approx C_{\mu}^{1 / 4} \sqrt{k_{P}}$ in the logarithmic region, as reminded below in eq. (25). Note that this expression is valid for boundary layers over smooth surfaces but it can be extended to rough surfaces since the velocity profile is simply shifted, still having a logarithmic behavior.

To preserve a viscous sublayer for smooth walls where $\mu_{t} \ll \mu$, a distance $y_{v}$ is introduced such that:

$$
\mu_{t}=\max \left(0, \alpha \mu\left(y^{*}-y_{v}^{*}\right)\right)
$$

with constant $\alpha=\kappa C_{\mu}^{1 / 4}$. Bidimensional boundary layer equations reduce to:

$$
\begin{aligned}
& \frac{\partial}{\partial y^{*}}\left[\left(\mu+\mu_{t}\right) \frac{\partial u}{\partial y^{*}}\right]=\frac{\nu^{2}}{k_{P}}\left[\frac{\partial}{\partial x}(\rho u u)+\frac{\partial P}{\partial x}\right]=C_{u} \\
& \frac{\partial}{\partial y^{*}}\left[\left(\frac{\mu}{P r}+\frac{\mu}{P r_{t}}\right) \frac{\partial T}{\partial y^{*}}\right]=\frac{\nu^{2}}{k_{P}}\left[\frac{\partial}{\partial x}(\rho u T)+S_{T}\right]=C_{T}
\end{aligned}
$$


with $S_{T}$ the averaged energy source term over the cell P. Considering $C_{u}$ and $C_{T}$ as constants, and using eq. (13) for the eddy viscosity $\mu_{t}$, analytical expressions for velocity and temperature profiles can be easily reached, since we have:

$$
\begin{aligned}
\frac{\mathrm{d} u}{\mathrm{~d} y^{*}} & =\frac{C_{u} y^{*}+A_{u}}{\mu+\mu_{t}} \\
\frac{\mathrm{d} T}{\mathrm{~d} y^{*}} & =\frac{C_{T} y^{*}+A_{T}}{\frac{\mu}{P r}+\frac{\mu_{t}}{P r_{t}}}
\end{aligned}
$$

Wall shear stress $\tau_{w}$ and wall heat flux $\phi_{w}$ have analytical expressions:

$$
\begin{aligned}
\tau_{w} & =\left(\mu+\mu_{t}\right) \frac{\mathrm{d} u}{\mathrm{~d} y}=\frac{\rho \sqrt{k_{P}} A_{u}}{\mu} \\
\phi_{w} & =-\left(\frac{\mu}{P r}+\frac{\mu_{t}}{P r_{t}}\right) \frac{\mathrm{d} T}{\mathrm{~d} y}=-\frac{\rho C_{p} \sqrt{k_{P}} A_{T}}{\mu}
\end{aligned}
$$

Integration constants $A_{u}$ and $A_{T}$ are determined by applying boundary conditions. Details are given in the appendix of Suga et al.paper [3]. Associated with these models for friction and heat transfer at the wall, production and dissipation terms for the turbulent kinetic energy are modeled as follows:

$$
P_{k}=\left\{\begin{array}{l}
0 \text { for } y^{*}<y_{v}^{*} \\
\nu_{t}\left(\frac{\mathrm{d} u}{\mathrm{~d} y}\right)^{2}=\frac{\alpha k_{P}}{\nu}\left(y^{*}-y_{v}^{*}\right)\left(\frac{C_{u} y^{*}+A_{u}}{\mu+\mu_{t}}\right)^{2} \text { for } y^{*} \geq y_{v}^{*}
\end{array}\right.
$$

and

$$
\varepsilon= \begin{cases}\frac{2 \nu k_{P}}{y_{\varepsilon}^{2}} & \text { if } y<y_{\varepsilon} \\ \frac{k_{P}^{3 / 2}}{c_{l} y} & \text { if } y \geq y_{\varepsilon}\end{cases}
$$


where a dissipation scale $y_{\varepsilon}$ is defined [12] through $y_{\varepsilon}^{*}=2 c_{l}$, with $c_{l}=$ 2.55. Mean values are computed through integration over the cell. To stay consistent with Suga et al. notations, $y_{n}$ will designate the distance to the wall cell center $P$. Integration over $\left[0, y_{n}\right]$ relates $\overline{P_{k}}$ to $A_{u}$ and $C_{u}$ coefficients. Several analytical expressions are provided by Suga et al. [3] regarding the relative value of $y_{v}$ with respect to $h$ and $y_{n}$ values. For $\bar{\varepsilon}$ the integration leads to:

$$
\bar{\varepsilon}= \begin{cases}\frac{2 k_{P}^{2}}{\nu y_{\varepsilon}^{* 2}} & \text { if } y_{n}^{*}<y_{\varepsilon}^{*} \\ \frac{k_{P}^{2}}{\nu y_{n}^{*}}\left(\frac{2}{y_{\varepsilon}^{*}}+\frac{1}{c_{l}} \ln \left(\frac{y_{n}^{*}}{y_{\varepsilon}^{*}}\right)\right) & \text { if } y_{n}^{*} \geq y_{\varepsilon}^{*}\end{cases}
$$

In the original model of Craft et al. [12] a numerical optimization on smooth flat plate configurations yields $y_{v}^{*}=10.7$.

To reproduce the shift $\Delta u^{+}$due to the roughness effect on velocity profiles, Suga et al. [3] allowed $y_{v}^{*}$ to vary with respect to the equivalent sand grain height $h_{s}$. When $h_{s}$ increases, $y_{v}$ must decrease to provide positive values of the eddy viscosity at the wall $\mu_{t_{w}}$. Therefore the wall friction $\tau_{w}=\left(\mu+\mu_{t}\right) \frac{\partial u}{\partial y}$ increases with $h_{s}$ as expected. The resultant function now denoted $y_{v_{r}}$ is:

$$
\begin{aligned}
& y_{v_{r}}^{*}=y_{v}^{*}\left(1-\left(\frac{h_{s}^{*}}{70}\right)^{m}\right) \\
& m=\max \left(\left(0.5-0.4\left(\frac{h_{s}^{*}}{70}\right)^{0.7}\right),\left(1-0.79\left(\frac{h_{s}^{*}}{70}\right)^{-0.28}\right)\right)
\end{aligned}
$$

Similarly to Aupoix's approach [2] for the thermal correction (10), Suga et al. also introduced a variable turbulent Prandtl number and proposed to write: 


$$
\begin{gathered}
\Delta \operatorname{Pr}_{t}=\mathcal{F}^{S} \max \left(0,1-\frac{y^{*}}{h_{s}^{*}}\right) \\
\mathcal{F}^{S}=\frac{5.5}{1+\left(\frac{h_{s}^{*}}{70}\right)^{6.5}}+0.6
\end{gathered}
$$

Contrary to Aupoix's correction (11), only $h_{s}$ is considered as a parameter for this turbulent Prandtl number correction.

The AWF of Suga [3] including roughness corrections has been validated over various boundary layer cases, ranging from standard flat plates to more complex flows such as curved walls or separating flows. For consistency reasons regarding Aupoix's work, it is nevertheless interesting to derive a new formulation of the AWF relying on principles exposed in section 2. The ultimate objective is to dispose of a unique turbulence model capable of working on both fine and coarse meshes and dealing with rough walls.

\section{Modified AWF}

Starting from the idea of Aupoix to use a wall shift $y_{0}$ according to eq. (5), a new model for $y_{v_{r}}$ has been build. In order to get a general expression for $y_{0}$, not related to any specific turbulence model contrary to Aupoix LRN approach that is restricted to the $k-\omega$ turbulence model, Reichardt's law [19] for a ZPG boundary layer velocity profile over a smooth surface is considered:

$$
u_{s}^{+}=\frac{1}{\kappa} \ln \left(1+\kappa y^{+}\right)+7.8\left[1-\exp \left(-\frac{y^{+}}{11}\right)-\left(\frac{y^{+}}{11}\right) \exp \left(-0.33 y^{+}\right)\right]
$$

From this profile, the shift $y_{0}^{+}$is computed with respect to $h_{s}^{+}$. A fitting function is then determined, it reads: 


$$
y_{0}^{+}=\left(0.0202 h_{s}^{+}+10.1\right) \tanh \left(\left(\frac{h_{s}^{+}}{90}\right)^{0.55}\right)
$$

In the inner region of the boundary layer the eddy viscosity can be expressed as $\mu_{t}^{+}=\left(\frac{\partial u^{+}}{\partial y^{+}}\right)^{-1}-1$. Reichardt's profile directly provides $\mu_{t}^{+}\left(y_{0}^{+}\right)=\mu_{t_{w}}^{+}\left(h_{s}^{+}\right)$. Since $y_{v_{r}}^{*}=-\frac{\mu_{t_{w}}^{+}}{\alpha}$, discretized values of $y_{v_{r}}^{*}$ are easily derived. Fitting functions are then build to match with the obtained curve.

$$
\begin{aligned}
& y_{v_{r}}^{*}=-\frac{1}{\alpha} f_{1}\left(h_{s}^{+}\right) f_{2}\left(h_{s}^{+}\right) f_{3}\left(h_{s}^{+}\right) f_{4}\left(h_{s}^{+}\right)+y_{v}^{*} \exp \left(-2 h_{s}^{+}\right) \\
& f_{1}=\left(\frac{h_{s}^{+}}{180}\right)^{1.15} \\
& f_{2}=\left[1+2 \exp \left(-\left(\frac{h_{s}^{+}}{100}\right)^{0.9}\right)\right] \\
& f_{3}=\left[1-0.4 \exp \left(-\left(\frac{h_{s}^{+}}{100}\right)^{1.2}\right)\right] \\
& f_{4}=\left(1+\ln \left(h_{s}^{+-0.9}\right)\right) \exp \left(-\frac{h_{s}^{+}}{7}\right)
\end{aligned}
$$

Function $f_{1}$ reproduces the global trend. Function $f_{2}$ improves the interpolation for $h_{s}^{+}$of order $\mathcal{O}(100)$ while functions $f_{3}$ and $f_{4}$ acts on $h_{s}^{+}$values of order $\mathcal{O}(10)$. The last term of eq.( 24) serves as a recovery term for smooth configurations.

The expression (20) for $y_{v_{r}}$ provided by Suga et al. depends on $y^{*}$ whereas the present expression of eq. (24) depends on $y^{+}$. To compare the two expressions, it is convenient to be able to switch from the Reynolds number $y^{*}$ to the standard nondimensional distance $y^{+}$. They are related by: 


$$
y^{*}=\frac{y \sqrt{k_{P}}}{\nu}=\frac{y u_{\tau}}{\nu} \frac{\sqrt{k}}{u_{\tau}}=y^{+} \sqrt{k^{+}}
$$

Bradshaw's relation gives:

$$
y^{*}=\frac{y^{+}}{\sqrt{a_{1}}} \quad k^{+}=\frac{1}{a_{1}} \quad a_{1}=0.3
$$

but it is only valid in the logarithmic region, i.e. $y^{+}>100$. To account for the whole inner region, the $k^{+}$profile obtained with the $k-\omega$ SST model has been modeled as:

$$
k^{+}=\frac{1}{a_{1}} \tanh \left(0.005 y^{+^{2}}\right)
$$

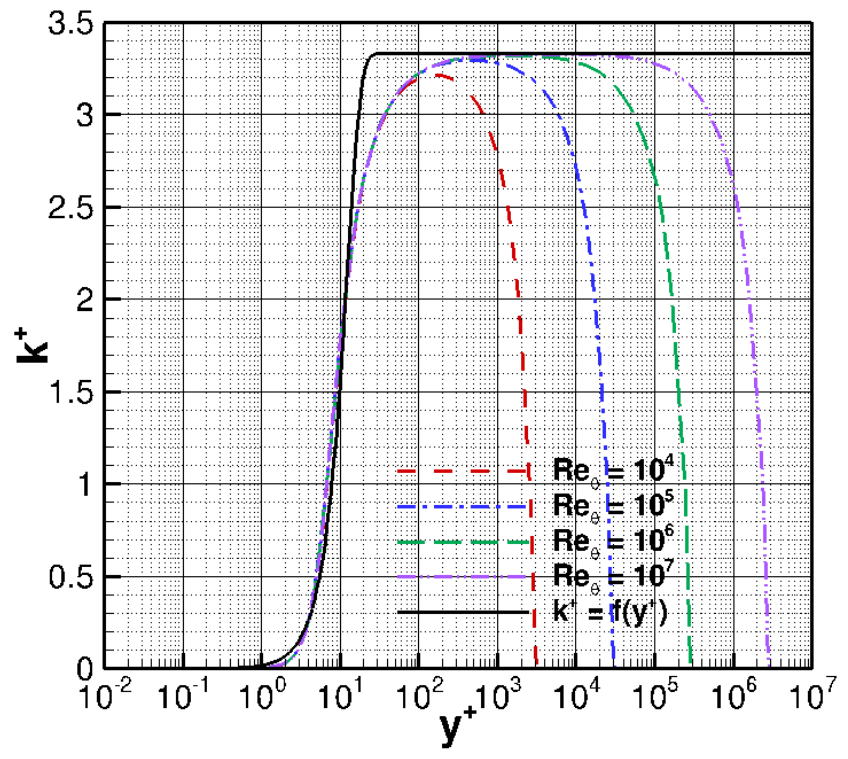

Figure 1: Turbulent kinetic energy profiles $k^{+}$of ZPG boundary layers. 
On figure 1 several turbulent kinetic energy profiles have been plotted. They have been obtained with the boundary layer code CLICET with the $k-$ $\omega$ SST model and correspond to solutions at various $R_{\theta}$ values, $R_{\theta}$ being the Reynolds number based upon the momentum. Relation (27) is an acceptable approximation in the inner region of boundary layers.

Using the model of the $k^{+}$profile provided by eq. (27) to switch from $y^{*}$ to $y^{+}$and vice versa, comparisons of $\mu_{t_{w}}$ expressions obtained by Suga et al. and given by eq. (24) are plotted on figures $2(\mathrm{a})$ and $2(\mathrm{~b})$.

Aupoix's model obtained from eq. (9) through $\mu_{t_{w}}^{+}=\frac{k_{w}^{+}}{\omega_{w}^{+}}$is also drawn on these figures as a reference. Important differences exist between the different models. Discrepancies existing between Aupoix LRN model and the present AWF model for $\mu_{t_{w}}^{+}$come from the different behaviors of Reichardt's profile and the $k-\omega \mathrm{SST}$ solution in the buffer region. For $h_{s}^{+} \in[100,1000], y_{0}^{+}$ values ranges from 10 to 30 . For a given altitude in the buffer layer, the differences existing in the $u^{+}$profile obtained from Reichardt's profile and the $k-\omega$ SST model, will lead to discrepancies in the evaluation of $\Delta u^{+}$. Finally, after introducing $h_{s}^{+}$thanks to eq. (2), rather large differences appear in the final form of $\mu_{t_{w}}^{+}$. This explains why, even though the same principles are applied between Aupoix's work and the present one, quite large differences exist on figure 2(a) and 2(b). As mentioned earlier, the present AWF model is designed to be used in conjunction with any turbulence model, justifying the use of the Reichardt's profile.

For $h_{s}^{*}$ values larger than 150, figure 2(a) shows that Suga's model yields larger slopes than the Aupoix LRN approach and the present model for $\mu_{t_{w}}$. The slope being much larger for Suga's model, differences are emphasized as 


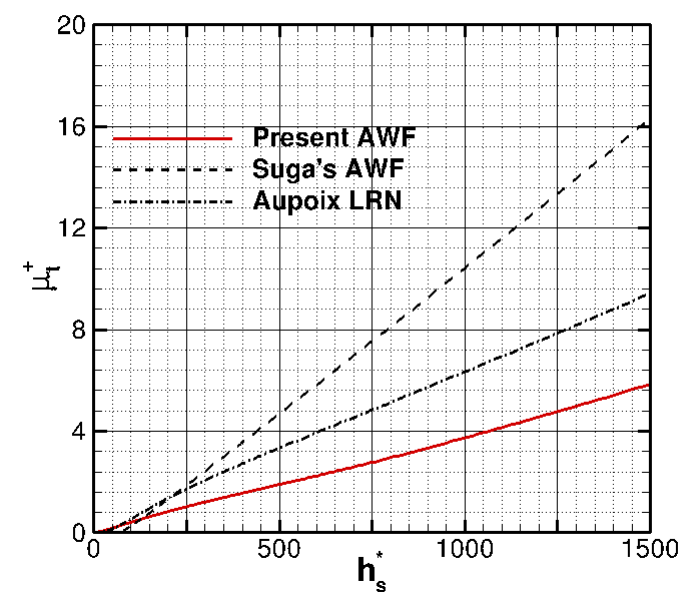

(a) $\mu_{t_{w}}^{+}=f\left(h_{s}^{*}\right)$

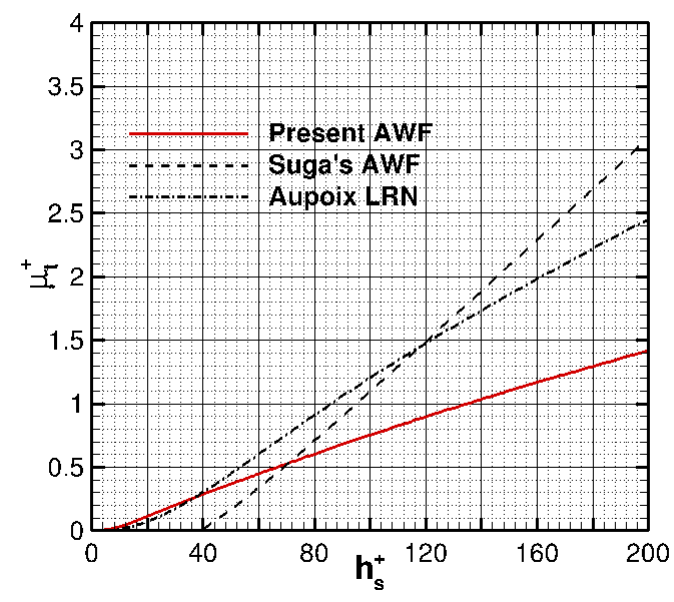

(b) $\mu_{t_{w}}^{+}=f\left(h_{s}^{+}\right)$

Figure 2: Eddy viscosity at the wall with respect to nondimensional equivalent sand grain heights $h_{s}^{*}$ and $h_{s}^{+}$. 
$h_{s}^{*}$ increase. On the contrary, for low $h_{s}^{+}$values, i.e. below 70 , the model proposed by Suga et al. exhibit lower values than the present AWF as shown on figure $2(\mathrm{~b})$. The threshold value $h_{s}^{+}=70$ corresponds incidentally to the entrance into the full rough regime without any obvious explanation.

The introduction of the wall shift $y_{0}^{+}$in the present model involves a modification of the averaged production term $\overline{P_{k}}$ and of the averaged dissipation rate $\bar{\varepsilon}$. For $\overline{P_{k}}$ the lower limit of integration must be shifted, see Appendix $\mathrm{B}$ for example, whereas for the dissipation rate $\bar{\varepsilon}$ it comes:

$$
\bar{\varepsilon}=\left\{\begin{aligned}
\frac{2 k_{P}^{2}}{\nu y_{\varepsilon}^{* 2}} & \text { if } y_{n}^{*}<y_{\varepsilon}^{*} \\
\frac{k_{P}^{2}}{\nu\left(y_{n}^{*}-y_{0}^{*}\right)}\left[\frac{2\left(y_{\varepsilon}^{*}-y_{0}^{*}\right)}{y_{\varepsilon}^{* 2}}\right. & \\
\left.\quad+\frac{1}{c_{l}} \ln \left(\frac{y_{n}^{*}}{y_{\varepsilon}^{*}}\right)\right] & \text { if } y_{n}^{*} \geq y_{\varepsilon}^{*} \geq y_{0}^{*} \\
\frac{k_{P}^{2}}{\nu\left(y_{n}^{*}-y_{0}^{*}\right) c_{l} \ln \left(\frac{y_{n}^{*}}{y_{0}^{*}}\right)} & \text { if } y_{n}^{*} \geq y_{0}^{*} \geq y_{\varepsilon}^{*}
\end{aligned}\right.
$$

To stay consistent with the previous formulation of the wall function established by Suga et al., the dissipation term of the turbulent kinetic energy in the wall cell is still expressed using $\bar{\varepsilon}$ in eq. (28). However, the present formulation will be compared to the LRN approach of Aupoix and therefore is meant to be used with the $k-\omega$ SST turbulence model. The dissipation term in the $k$-equation reads $C_{\mu} k \omega$ and consequently $\bar{\omega}=\frac{\bar{\varepsilon}}{C_{\mu} k_{P}}$. Regarding the thermal correction, and relying on the modified Prandtl number given by eq. (11), Suga's model is revisited. In order to ease integral 


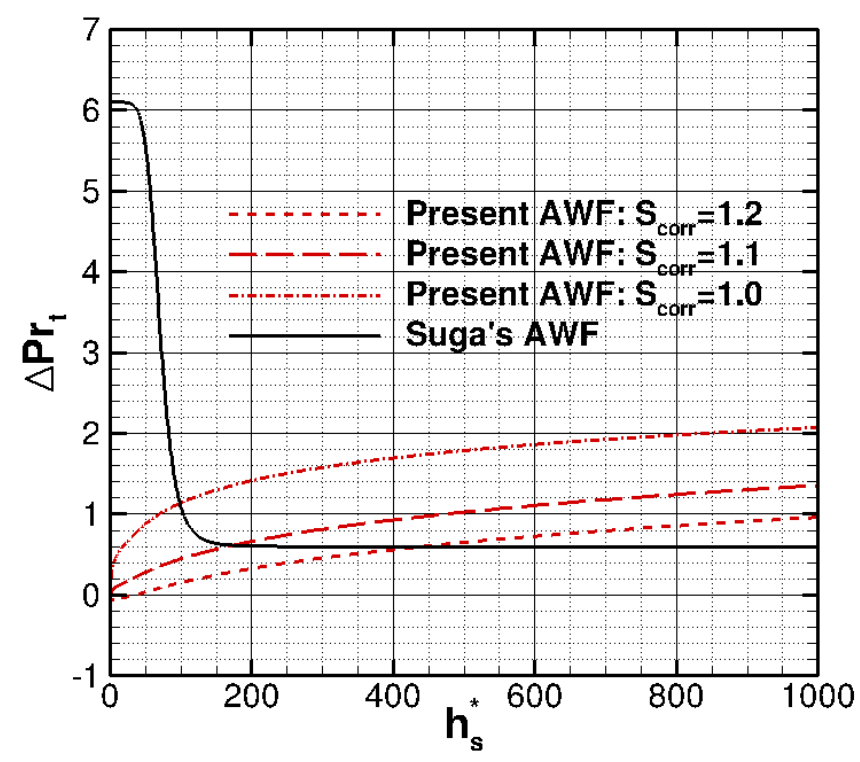

Figure 3: Thermal correction $\Delta P r_{t_{w}}$ at the wall

calculations, the initial exponential behavior is altered as follows:

$$
\Delta P r_{t}=\mathcal{F}^{A}\left(h_{s}^{+}, S_{c o r r}\right) \max \left(0,1-\frac{y^{*}}{a h_{s}^{*}}\right)
$$

with $a=1.3 h / h_{s}$. Details on the impact of $a$ on the analytical form of heat transfer are given in Appendix A.

As pointed out in section 3, Suga's thermal correction only depends on $h_{s}^{*}$ whereas three parameters take part in eq. (29), since $\mathcal{F}^{A}$ depends on $h_{s}^{+}$ and $S_{c o r r}$. To illustrate and highlight differences that exist between the two proposed turbulent Prandtl number modifications, corrections $\left.\Delta P r_{t}\right|_{y=0}=$ $\Delta P r_{t_{w}}$ at the wall are depicted on figure 3. For the present wall function, three curves are plotted to highlight the influence of the corrected wetted surface ratio $S_{\text {corr }}$. Although large discrepancies exist for low values of $h_{s}^{+}$, 
the values of $\Delta P r_{t_{w}}$ provided by Suga's model should be analyzed carefully in this range. For $h_{s}^{*}<70$, Suga's model provides $y_{v_{r}}>0$ leading to $\mu_{t_{w}}=0$. The turbulent thermal conductivity at the wall $\lambda_{t_{w}}$ will be zero regardless of the value of the turbulent Prandtl number correction $\Delta P r_{t_{w}}$. However, although the whole thermal correction over the wall cell may not be zero, it is still low for $h_{s}^{*} \in[0,70]$ due to the damping imposed in eq.( 21). Nevertheless, beyond the effect of $S_{\text {corr }}$, for large values of $h_{s}^{+}$major differences appear in the behavior of $\Delta P r_{t_{w}}$ between Suga's model and the one presented in this paper.

\section{Implementation and validation procedure}

The original corrections proposed by Aupoix [1, 2] and both AWF formulations presented in section 3 and 4 have been implemented in the CEDRE code [20] developed at ONERA. The CEDRE code is designed to perform massively parallel computations and is devoted to multi-physic configurations. This code contains several solvers among which the main one is a compressible reactive Navier-Stokes solver called CHARME. It uses generalized unstructured meshes that necessitate modifications in order to be able to implement the AWF models. Only local information is available in each cell whereas the thermal correction (11) requires information related to the wall faces in all cells of the fluid domain. A dedicated data structure was added to allow information exchange from wall faces to volume cells. Finally,

for each wall boundary condition, users are asked to input values for $h_{s}, h$ and $S_{c o r r}$ if necessary. In parallel, wall functions can be activated on demand. 
In the framework of the development of their AWF, Craft et al. assume the use of hexahedral or quadrangle cells. Here, the requirement of the CEDRE code and the possible use of polyhedral cells force changes in the definition of $y_{n}$. It no longer refers to the "north" face of the wall cell $P$ but to the location of the cell center. Figure 4 illustrates this modification compared to the original AWF.

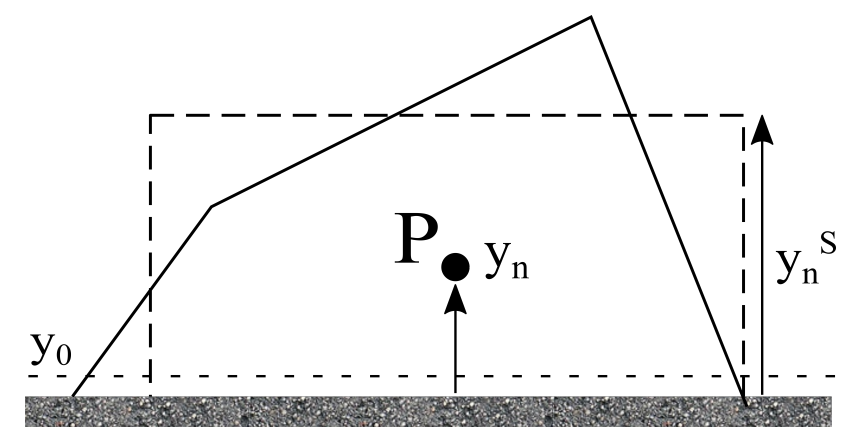

Figure 4: Definition of the wall distance $y_{n}$ for a triangle cell. Dashed quad stands for the equivalent cell used to define the wall distance in Suga's model $y_{n}^{S}$.

Integral calculations for $\bar{P}$ and $\bar{\omega}$ are performed over the interval $\left[y_{0}, y_{n}\right]$ but results are applied at cell centers i.e. at $y=y_{n}$. Since mean values are assumed constant within integral bounds it could be applied anywhere in the range $y \in\left[y_{0}, y_{n}\right]$. The convective terms and the pressure gradients parallel to the wall are assumed constant over the wall cells and are set equal to their values at wall cell centers. The same technique applied to a wall function resolving a 1D boundary layer problem [21] was already proved to give satisfactory results.

The changes in the definition of $y_{n}$ and the use of a conventional ap- 
proach to treat wall-parallel convective fluxes may render the present AWF less predicitive in flows involving separations. Additional work is probably required to improve the behavior of the present model in presence of such flows. In the validation section, configurations are therefore intentionally restricted to attached boundary layer configurations.

Finally, the Navier-Stokes solver CHARME also requires a value for $\omega_{n}$ in order to evaluate turbulent scalar fluxes at the wall. $\omega_{n}$ differs from $\bar{\omega}$ which is the mean specific dissipation in the wall cell whereas $\omega_{n}$ only serves to evaluate fluxes at the wall. By construction, $\bar{\omega}$ corresponds to an approximation of $\omega$ at location $\frac{y_{n}-y_{0}}{2}$. As the turbulent kinetic energy $k_{P}$ is assumed constant in the wall cell and since in the turbulent region, $\varepsilon$ is usually taken as proportional to the inverse distance, the following expression for $\omega_{n}$ is retained:

$$
\omega_{n}=\frac{\bar{\varepsilon}\left(y_{n}-y_{0}\right)}{2 y_{n} C_{\mu} k_{P}} \approx \frac{\bar{\varepsilon}}{2 C_{\mu} k_{P}} \text { as long as } y_{0} \ll y_{n}
$$

Before presenting validation cases, some of the choices made have to be discussed. First, the retained validation cases present large $h_{s}^{+}$values. In the majority of the computations performed, $y_{n}^{+}$is lower than $h_{s}^{+}$. There is no contradiction in using $y_{n}$ values lower than $h_{s}$ but this particular case was not treated by Suga et al. in their AWF formulation [3]. In Appendix B details are given on the expressions to be adopted for such cases. As a consequence, the thermal correction of the AWF models may not be entirely contained in the wall cell. Therefore, the model must not be used without considering a complementary correction acting in all cells above, otherwise heat transfers would be badly captured. For this reason, Aupoix's correction (11) is acti- 
vated in all the following computations. AWF models are active in wall cells whereas Aupoix's thermal correction is only activated in the other cells. This is obviously in line with the idea of deriving a new wall functions relying on principles used by Aupoix $[1,2]$. Second, the modification in the definition of $y_{n}$ was made to deal with any kind of unstructured mesh. However, to capture a boundary layer properly prisms layers are generally used at walls. For bidimensionnal boundary layer flows, wall cells are thus quad cells. For this reason, all the meshes for the validation cases of section 6 make use of quad cells. At last, a clear distinction must be made between the extension of the AWF proposed by Suga et al. and the present implementation including the necessary modifications listed above. In section 6, comparisons only highlight the differences on the formulations used by Suga et al. and the one presented in this work for $\mu_{t_{w}}$ and $\Delta P r_{t}$. No direct conclusion can be drawn about the extension of the AWF by Suga et al. from the present results as the implementation in the unstructured code of the CEDRE plateform induced important changes. In the following, Suga's AWF refers only to the wall function present implementation associated with Suga's closure for $\mu_{t_{w}}$ and $\Delta P r_{t}$.

\section{Validation}

The models developed by Aupoix have been extensively validated over a large variety of boundary layer flows over rough surfaces. Similarly, the AWF formulation of Craft et al. and further developments of Suga et al. have also been proven to provide good results on a variety of wall flows. However, because some modifications were made to the model of Suga, it is 
important to confirm the capacity of the present wall function formulation to reproduce roughness effects and also check mesh sensitivity. First, a ZPG adiabatic boundary layer configuration is computed. Then, two configurations highlighting the benefits of the present model compared to Suga's work are considered. Two specific points will be examined. As reminded in section 3, the thermal correction (21) developed by Suga et al. only depends on $h_{s}$, whereas the present work requires two additional parameter $h$ and $S_{\text {corr }}$. However, it is known that the wetted surface influences heat transfer on rough walls. Therefore, the second validation test case focuses on to the influence of the wetted surface on heat transfer since one of the improvement brought by the present model lies in the form of the thermal correction (29). Figure 2(a) showed that as $h_{s}^{*}$ becomes large, $y_{v_{r}}^{*}$ formulation (20) given by Suga et al. diverges from that of eq. (24). The last set of validation cases will thus consider boundary layer flows over rough walls for which reduced equivalent sand grain height $h_{s}^{+}$are known to be very large.

\section{1. zero pressure gradient boundary layer flows}

A boundary layer developing on a flat plate is considered with an external velocity $U_{e}=100 \mathrm{~m} / \mathrm{s}$. An adiabatic wall is imposed such that roughness effects only act on friction. Thus, a unique parameter, the equivalent sand grain height $h_{s}$, controls the roughness correction. For simplicity reasons, the thermal correction (29), depending on two additional parameters, will only be tested in the following validation cases. Two equivalent sand grain height $h_{s}$ have been retained for the computations. Corresponding $h_{s}^{+}$values are 165 and 692. Three meshes have been used. The first one is made to be used in conjunction with the LRN approach of Aupoix. For the two other 
meshes, near wall cell heights are $y_{n}=1.09 e^{-3} \mathrm{~m}$ and $y_{n}=5.45 e^{-4} \mathrm{~m}$ for mesh M1 and M2 respectively.

Figure 5(a) shows the mean velocity profiles expressed in wall variables. For each of the two $h_{s}$ values, the computations made using the three meshes are in good agreement. The maximum relative error between results obtained with the wall function approach and Aupoix's LRN approach is $2 \%$ in terms of friction velocity $u_{\tau}$ whereas the maximum difference between results obtained with meshes $M 1$ and $M 2$ does not exceed 1\%. In addition, all results correctly recovered the velocity shift $\Delta u^{+}$given by eq. (2) and illustrated by dashes lines on the figure.

Figure 5(b) compares turbulent kinetic energy profiles and dissipation profiles obtained in the computations. The good agreement between the LRN model of Aupoix and the present AWF validates the modifications made to Suga's model especially regarding the production term $\overline{P_{k}}$ and the dissipation term $\bar{\varepsilon}$ in eq. (17) and eq. (28).

The objective of the validation test cases is to focus on two aspects. First, since the evolutions of $\mu_{t_{w}}$ differ between the two AWF models for large values of $h_{s}^{+}$, it is necessary to determine which of the two formulations performs best. Second, the use of function $\mathcal{F}^{A}$ instead of $\mathcal{F}^{S}$, introducing the wetted surface as a parameter, raises the question of the interest of the proposed approach. 


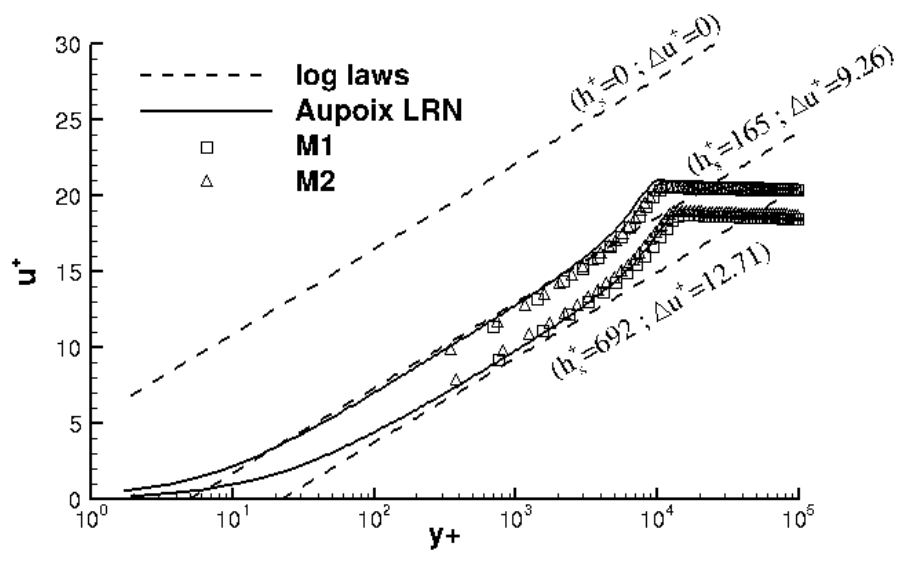

(a) velocity

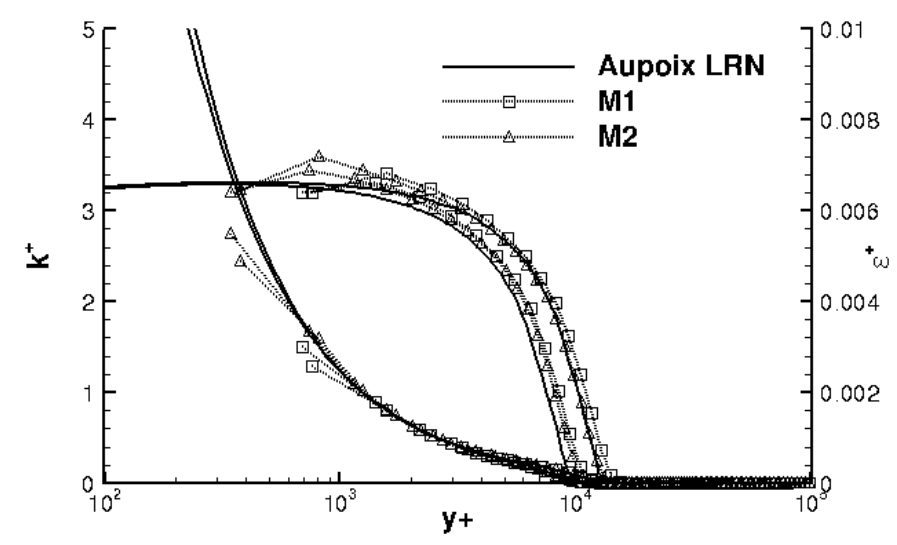

(b) turbulent scalars

Figure 5: Comparisons of dimensionless profiles obtained on a ZPG boundary layer. Full lines correspond to results obtained with the LRN model of Aupoix. Symbols represents results obtained with the new wall function on two differents meshes. Dashed lines are logarithmic laws provided by eq. (1) and eq. (2).

\subsection{Hosni et al. experiments}

At Mississippi State University, Hosni et al. [22] realized a series of experiments on rough surfaces. Among those, we here consider the flat plate 25 
experiments on which hemispheric roughness elements have been mounted in staggered rows. The distance $L$ from one element to another is $L / D=2$, with $D=1.27 \mathrm{~mm}$ the diameter of the hemispheres. For these experiments both friction coefficient and Stanton number distributions along the plate are available. The free-stream velocity retained for computations is $U_{e}=58.2 \mathrm{~ms}^{-1}$. Using the Waigh and Kind correlation [23] the equivalent sand grain height $h_{s}$ can be estimated at $1.58 \mathrm{~mm}$ while $S_{\text {corr }}$ is found to be 1.17 and $h=D / 2$.

To investigate the effects of the wall cell height on the two AWF formulations, several meshes have been considered. All meshes are composed of quads but are treated as unstructured meshes by the CEDRE code. All meshes use the same longitudinal points distribution along the $x$-axis. In the following $m$ will designate the number of points in the $y$-direction normal to the wall. Figure 6 gives an illustration of the point distribution along the vertical $y$-axis in the vicinity of the wall.

The fine mesh uses $m=185$ whereas meshes M1 to M4 respectively use $m=\{100,75,90,60\}$. This leads to ratio $y_{n} / h_{s}$ respectively of 0.70 for mesh M1 and M2, 0.35 for mesh M3 and 1.40 for mesh M4. As the friction velocity $u_{\tau}$ evolves along the plate, $y_{n}^{+}$and $h_{s}$ values vary. Table 1 gives an overview of the $y_{n}^{+}$values encountered during computations, indicating that with $h_{s}=1.58 \mathrm{~mm}$ one obtains $h_{s}^{+}$values ranging from 265 up to approximately 550.

Results obtained on friction coefficient $C_{f}$ and Stanton number $S t$ are presented on figures $7(\mathrm{a}), 7(\mathrm{~b}), 8(\mathrm{a})$ and $8(\mathrm{~b})$ for both variants of the wall function. 


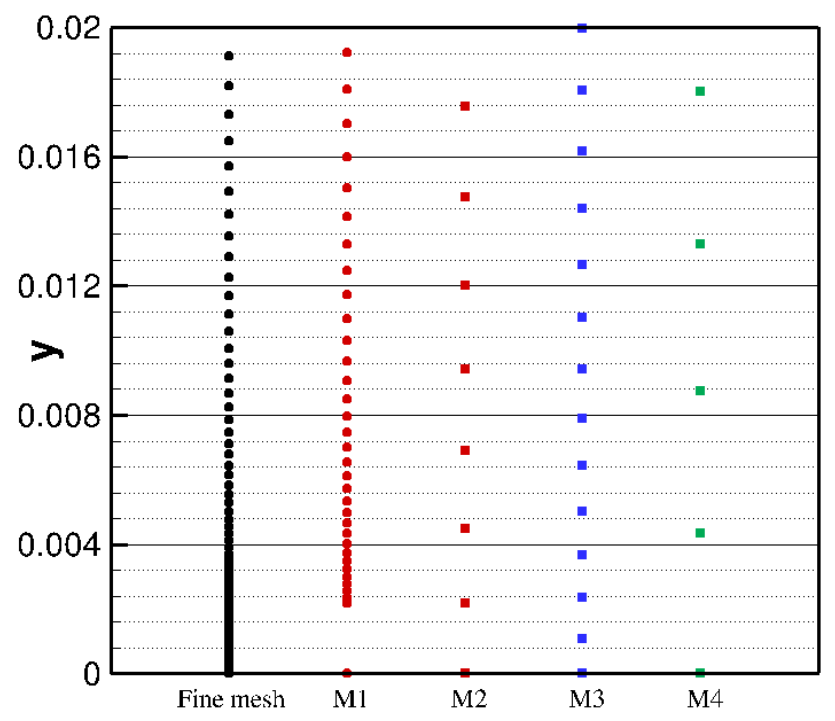

Figure 6: Points distribution along the $y$ axis of the 5 meshes used for computations of the MSU experiment

Remarkably, results are weakly dispersed with respect to the mesh used for both AWFs in the right hand side of the figure, i.e. in the developed part of the boundary layer. However, one can note that all computations on coarse meshes need a certain distance before recovering the good trend for $C_{f}$ and $S_{t}$ distributions. The coarser the mesh, the longer the recovery distance is. Accounting for convective terms in the AWFs would likely improve the results on that point. If Suga's AWF formulation provides a better agreement regarding friction coefficients, the present AWF results tend to be closer to experiments for heat transfers. In addition, $C_{f}$ coefficients given by the present AWF are in good agreement with the results of the model of Aupoix obtained with the fine mesh. Given the experimental uncertainty, it is hard 


\begin{tabular}{|c|c|c|}
\hline & $\min \left(y_{n}^{+}\right)$ & $\max \left(y_{n}^{+}\right)$ \\
\hline M1 & 187 & 389 \\
M2 & 188 & 390 \\
M3 & 93 & 190 \\
M4 & 375 & 680 \\
\hline
\end{tabular}

Table 1: Minimum and maximum values of $y_{n}^{+}$obtained with the present AWF for the four coarse meshes used to compute Hosni et al. experiments

to claim that one of the two AWF works better: they both perform well.

At location $x=1.68 \mathrm{~m}$, tangential velocity profiles $u$ are extracted and plotted in wall variables on figure 9. Experimental profiles [24] are plotted twice with respect to $y^{+}$and $y^{+}-y_{w}^{+}$. Here $y_{w}^{+}$stands for the fictitious origin of the wall distances for roughness elements placed over a flat plate. Under a certain distance from the wall, there is no contribution of the flow to the drag forces: $y_{w}^{+}$stands for this distance. Jackson [25] proposes to define $y_{w}$ as the point of application of the drag forces and indicates that $y_{w}$ lies between 0.5 and 0.84 times the height of the roughness elements. In figure $9 y_{w}$ is fixed at $y_{w}=0.84 h=0.5334 \mathrm{~mm}$ leading to $y_{w}^{+}=92.25$. Note that $y_{w}$ is not related to $y_{0}$.

Experimental velocity profiles plotted with respect to $y^{+}-y_{w}^{+}$permit to recover the log law slope on a larger range of $y^{+}$values as expected. LRN computations on the fine mesh and the profile obtained with the present AWF match the experimental profile well. Results from Suga's AWF are simply shifted due to the difference obtained on friction velocity $u_{\tau}$. This 


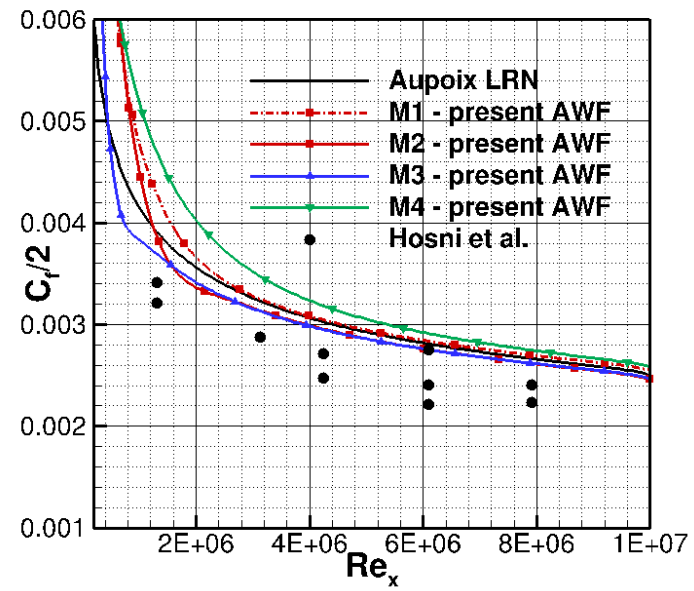

(a) friction coefficient

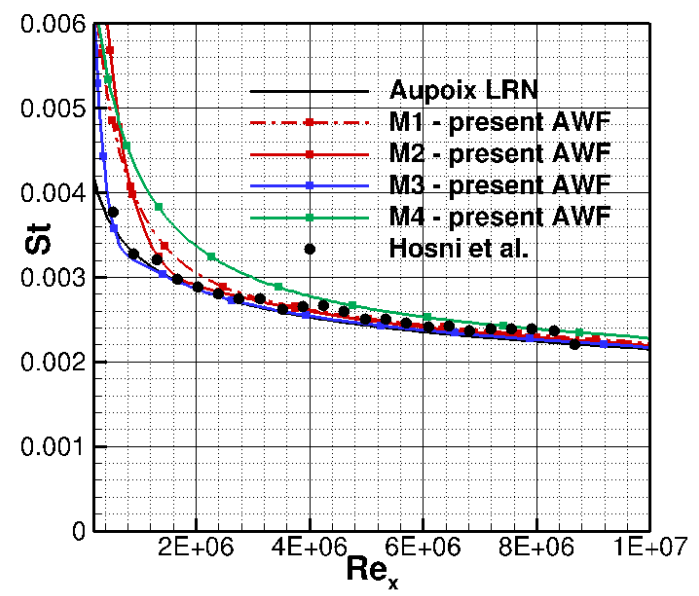

(b) Stanton number

Figure 7: Friction coefficient and Stanton number distributions obtained with the present AWF in the experimental configuration of Hosni et al. 


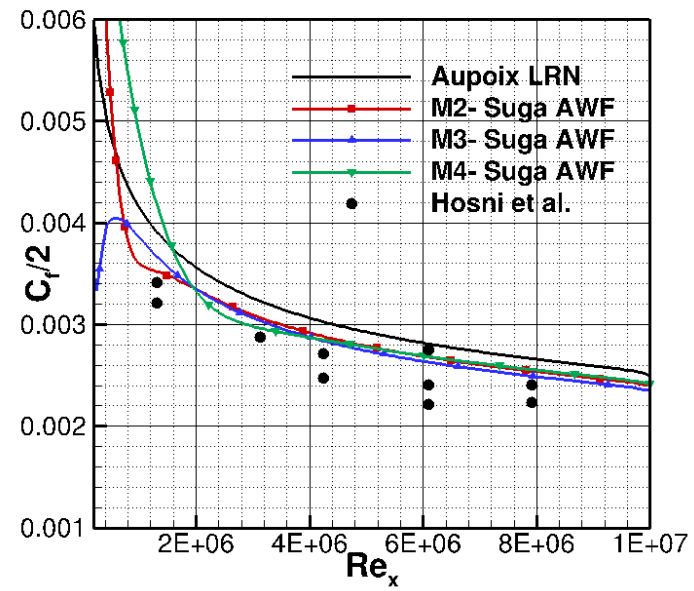

(a) friction coefficient

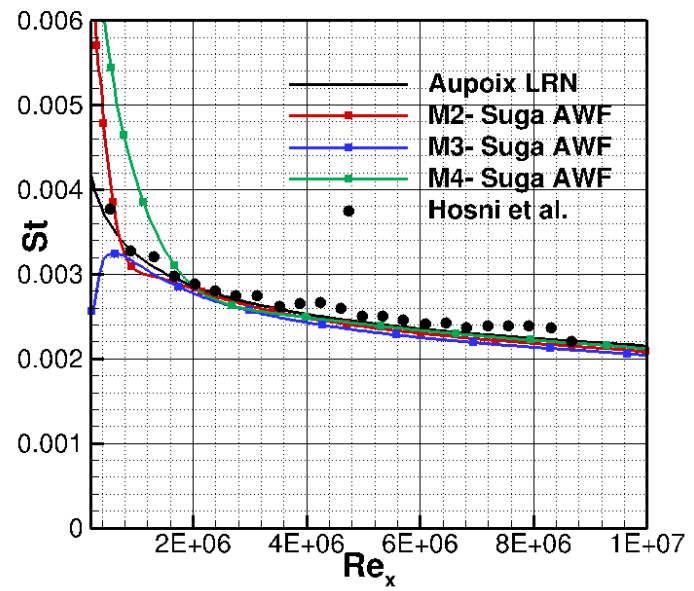

(b) Stanton number

Figure 8: Friction coefficient and Stanton number distributions obtained with Suga's AWF in the experimental configuration of Hosni et al. 


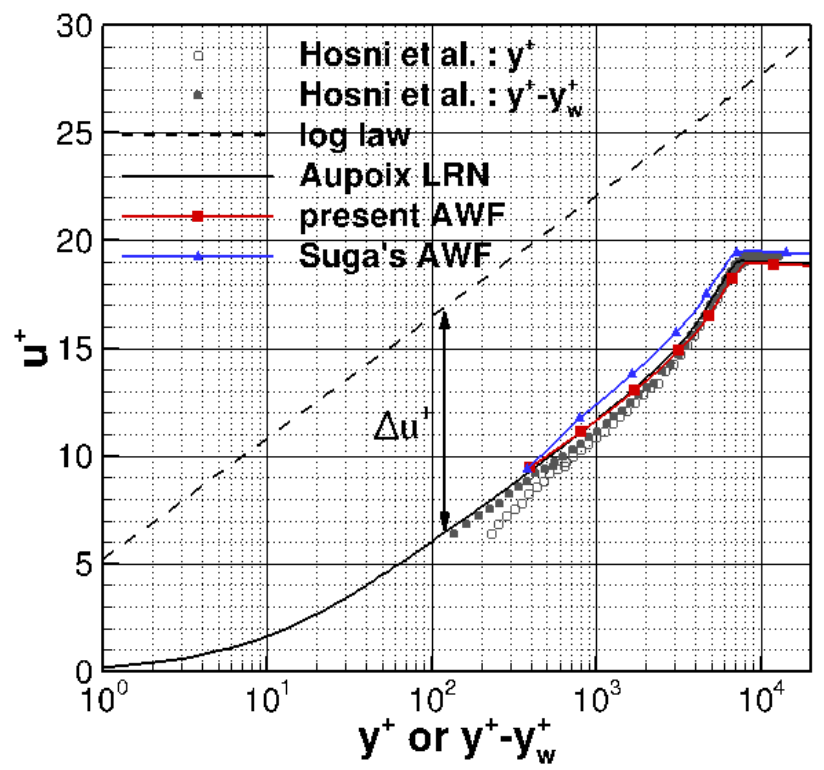

Figure 9: Comparisons of boundary layer profiles at location $x=1.68 \mathrm{~m}$. Measurements [24] correspond to a friction coefficient $C_{f}=0.0055$.

shift also appear at the edge of the boundary layer since $U_{e}^{+}=\sqrt{\frac{2}{C_{f}}}$. Finally, a slight bend is observed at the level of the near wall point on the profile obtained with Suga's AWF.

It is interesting to check the relevance of the Waigh and Kind correlation that leads to $h_{s}=1.58 \mathrm{~mm}$ from figure 9 . Experimental friction coefficient at $x=1.68 \mathrm{~m}$ is $C_{f}=0.0055$ leading to $h_{s}^{+}=\frac{h_{s} U_{e}}{\nu} \sqrt{\frac{C_{f}}{2}}=276$. Graphically one has $\Delta u^{+} \in[10.6,10.8]$ which provides $h_{s}^{+} \in[262,284]$ using eq. (2), proving that the estimation of $h_{s}$ is satisfactory.

In their experiments, Hosni et al. also investigated flat plate configu- 


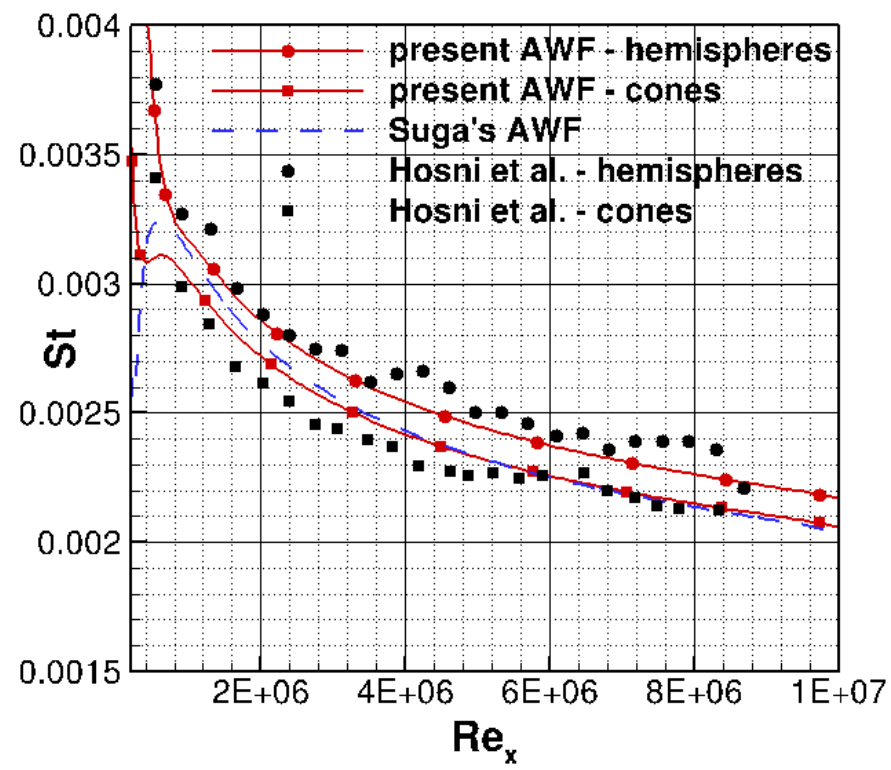

Figure 10: Effect of the wetted surface on Stanton numbers in Hosni et al. configurations for hemispheres and cones. All computations are made using mesh M3 and $h_{s}=1.58 \mathrm{~mm}$. The corrected wetted surface $S_{\text {corr }}$ is 1.17 and 1.09 respectively for hemispheres and truncated cones.

rations covered by truncated cones. These cones were designed in order to produce the same drag increase as the hemispheres. Their base diameter is $D$ and they are truncated at $h=D / 2$ with a half top angle of $40^{\circ}$. The spacing remains identical to the configuration with hemispheres. As truncated cones produce the same friction increase, they share the same equivalent sand grain height value $h_{s}$. However, their respective wetted surfaces differ and one finds $S_{\text {corr }}=1.09$ for the truncated cones. Therefore, despite a similar effect on friction, lower heat transfers are expected in this configuration compared to the one with hemispheres. 


\begin{tabular}{|c|c|c|c|}
\hline & $h_{s}[\mathrm{~mm}]$ & $h[\mathrm{~mm}]$ & $S_{\text {corr }}$ \\
\hline case 7 & 2.25 & 1.09 & 1.08 \\
case 2 & 2.94 & 2.87 & 1.18 \\
case 3 & 15.63 & 3.07 & 1.22 \\
\hline
\end{tabular}

Table 2: Values of parameters $h_{s}, h$ and $S_{\text {corr }}$ used for computating configurations tested by Dukhan et al..

Figure 10 presents experimental results obtained by Hosni et al. for configurations with hemispheres and cones. A significant drop in Stanton numbers is observed between the two tested plates. The present AWF results for mesh M3 are also reported on figure 10 to illustrate the ability of the new formulation of the Prandtl modification (29) to reproduce the effect of the wetted surface on heat transfer. A unique curve is available with Suga's model since it only depends on $h_{s}$ which remain the same for truncated cones and hemispheres. Even if the effect is slightly underestimated, the present AWF closure brings improvements to the model of Suga et al..

\subsection{Dukhan et al. experiments}

In the late 90's, ice shapes obtained in the NASA Icing Research Tunnel were copied and reproduced on flat plates by Dukhan et al. [26]. The singularity of these experiments is that some of the rough elements correspond to remarkably large values of the equivalent sand grain height. Here, we consider three cases numbered 2, 3 and 7 and corresponding to rime ice and rough glaze ice. Table 2 summarizes the different values of parameters characterizing the three test cases. 
Dirling's correlation [27] has been used to determine $h_{s}$ values. The corrected wetted surface ratio $S_{\text {corr }}$ is taken as the ratio between the wetted rough surface and the smooth surface without corrections. This parameter is limited to the maximum value for hemispheres, i.e. $S_{\text {corr }}=1.22$ for case 3 . The roughness inserts are mounted after an initial smooth region of $0.0508 \mathrm{~m}$ length. The free-stream velocity considered here is $47.24 \mathrm{~ms}^{-1}$. Only Stanton number values are available for these experiments. The different experimental conditions lead to large mean values of $h_{s}^{+}$, around 400 and 600 for cases 7 and 2 and up to 4000 for case 3. These configurations should allow the exploration of the limits of the AWF formulations for $\mu_{t_{w}}$ and $\Delta P r_{t}$.

Three meshes were used for all the computations. The first one is a fine mesh used for LRN computations. The second and third ones denoted M1 and M2 are coarse meshes with $y_{n}=2 \mathrm{~mm}$ and $y=4 \mathrm{~mm}$ respectively. All grids share the same longitudinal points distribution. Meshes M1 and M2 are composed of 50 and 20 nodes in the $y$ direction respectively whereas the fine mesh uses 130 points.

Figure 11(a), 12(a) and 13(a) compare the obtained results to the experimental data for the three configurations. The new AWF exhibits acceptable results, consistent with respect to the LRN computations and the measurements. As seen previously, a delay is observed before recovering the main trend. It is important to remind that in these configurations, $h_{s}^{+}$can be very large, especially at the onset of the boundary layer, and up to 20 times larger than $y_{n}^{+}$for mesh M1 in case 3. Figures 11(b), 12(b) and 13(b) provide the distributions of $y_{n}^{+}$and $h_{s}^{+}$values obtained with the present AWF model. 


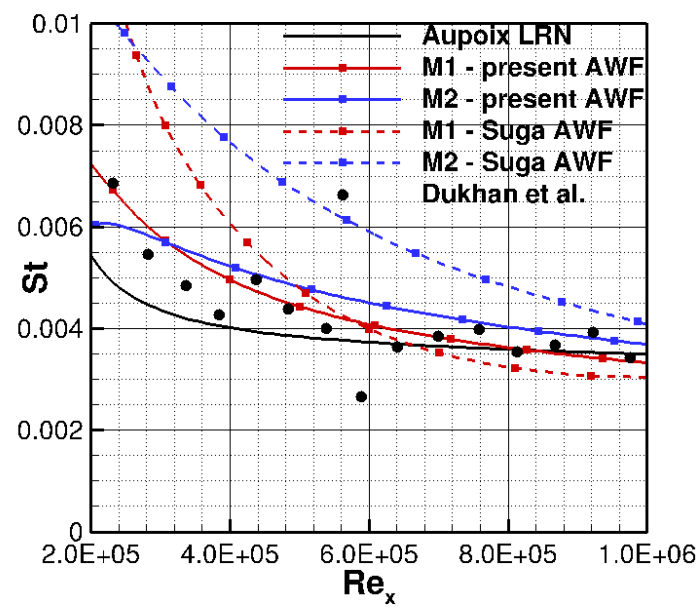

(a) Stanton number

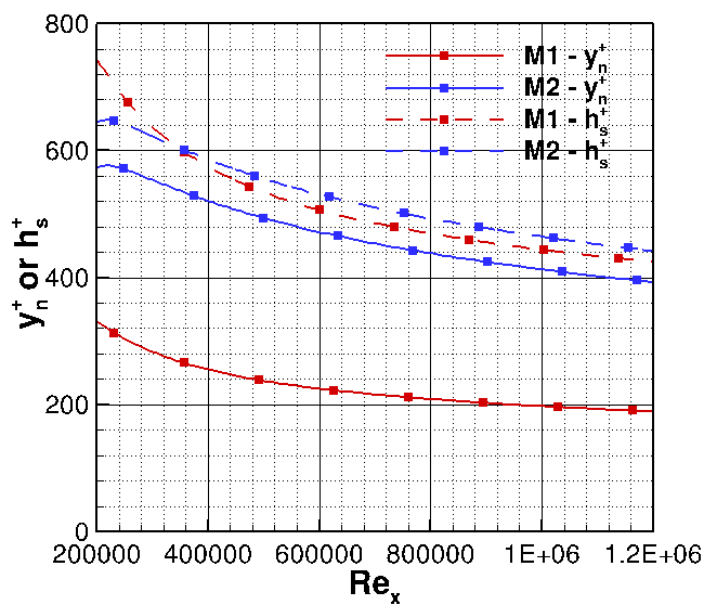

(b) nondimensional heights

Figure 11: Stanton number, $y_{n}^{+}$and $k_{s}^{+}$distributions along the plate for cases 7 of Dukhan et al. experiments. Nondimensional heights distributions are obtained from the present AWF model. 


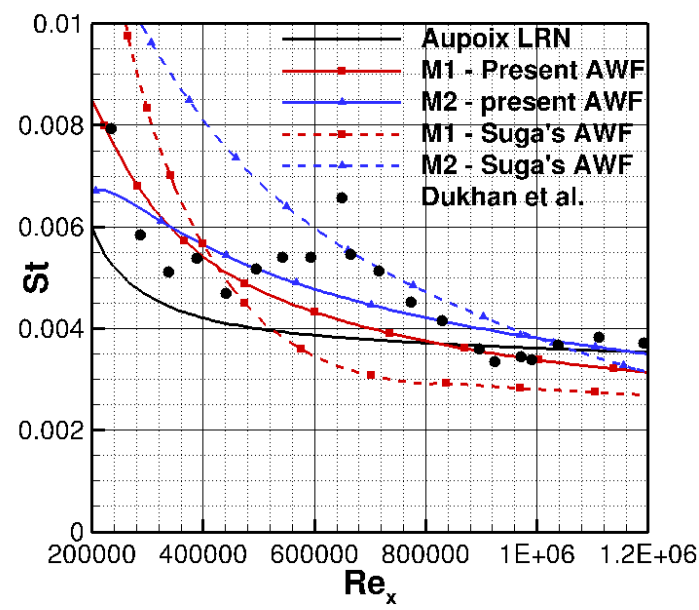

(a) Stanton number

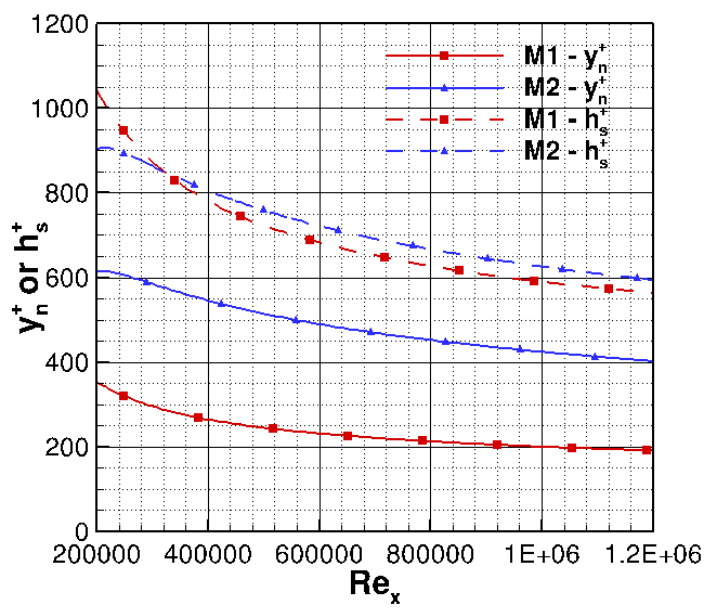

(b) nondimensional heights

Figure 12: Stanton number, $y_{n}^{+}$and $k_{s}^{+}$distributions along the plate for cases 2 of Dukhan et al. experiments. Nondimensional heights distributions are obtained from the present AWF model. 


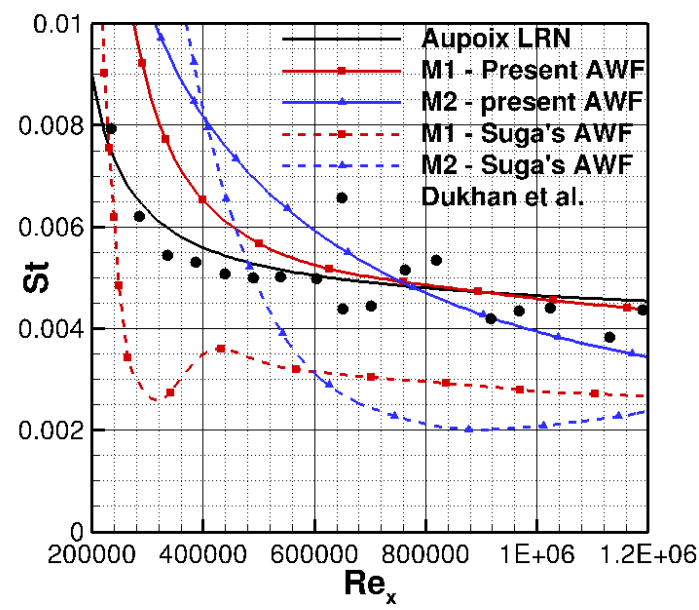

(a) Stanton number

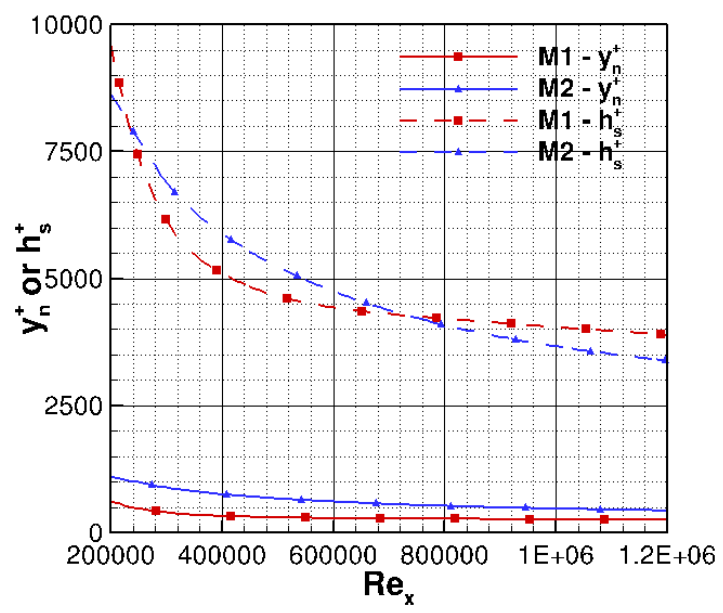

(b) nondimensional heights

Figure 13: Stanton number, $y_{n}^{+}$and $k_{s}^{+}$distributions along the plate for cases 3 of Dukhan et al. experiments. Nondimensional heights distributions are obtained from the present AWF model. 
Even though $k_{s}^{+}$values change for mesh M1 and mesh M2, the relative errors for $R e_{x}>3 e^{5}$ do not exceed $5 \%$ for case 7, $6.5 \%$ for case 2 and reach $15 \%$ case 3. Given the values of $y_{n}$ and $h_{s}$ encountered in these computations, this relative errors may be considered as acceptable. Regarding Suga's AWF, neither a good agreement with experimental data nor a low dispersion between results is observed. As mentioned above, since $y_{n}$ is smaller than $h_{s}$, the Prandtl turbulent number correction is not entirely included in the wall cells. Aupoix's correction is activated in cells above the wall cells to compensate. However, with Suga's model the combined use of the two thermal corrections does not permit to recover a good estimation of heat transfer. As alluded in section 5, without Aupoix's correction on the Prandtl numbers results are even worse. The validation test cases coming from Dukhan et al. data [26] demonstrate the benefits of the present AWF formulation in terms of ability to reproduce thermal effects due to roughness in configurations where $h_{s}^{+}$can be very large.

\section{Concluding remarks}

A modification to the analytical wall function able to account for roughness effects and developed by Suga et al. [3] was proposed in this paper. The objective of the proposed modification is to use the principles driving the rough wall corrections of Aupoix [1,2]. The present AWF formulation was compared to the initial model by Suga et al. and has been proven to enlarge its range of applications. The great interest of Suga's wall law is preserved and several additional benefits can be highlighted:

- The extension of the AWF proposed by Suga et al. was modified to 
be adapted to unstructured codes. Modifications in the definition $y_{n}$, see figure 4 , and its consequences on formulations of $\tau_{w}, \phi_{w}, \overline{P_{k}}$ and $\bar{\varepsilon}$ were made. For meshes including prisms layers at walls, the induced modifications were proved to work adequately.

- The effect of the increase of the wetted surface due to roughness is now considered in the formulation of the modified turbulent Prandtl number using parameter $S_{\text {corr }}$ in eq. (11).

- For large $h_{s}^{+}$, for which $y_{n}^{+}$may be lower to $h_{s}^{+}$, a consistent behavior is observed with the present AWF formulation for $\mu_{t_{w}}$ and $\Delta P r_{t}$. However, further tests are required to better characterize the consistency of the approach where $y_{n}^{+}<h_{s}^{+}$, especially in presence of pressure gradients.

Even if undeniable progress have been made with the present AWF, there are still some points that deserve further work. First, refinements developed in the original AWF by Craft et al. [12] could be adapted to the present model to improve its predictive capabilities. Additionally, the desirable objective to have a model relatively insensitive to mesh is not yet achieved. Modification of the mean dissipation rate $\bar{\varepsilon}$ using relation (9) for $\omega_{w}$ may allowed the AWF to degenerate into the LRN model of Aupoix [1] when $y_{n}^{+}$tends to 1. The difficult question of the buffer layer may also be treated using an ad-hoc function on $\bar{\varepsilon}$. In addition, if by construction the present AWF is not related to any specific turbulence model, it was considered to be used in conjunction with the roughness correction adapted to the $k-\omega$ SST model. A more general approach may be considered but will require the reformulation of 
Aupoix's corrections.

\section{Acknowledgments}

The author is extremely grateful to Bertrand Aupoix for sharing his knowledge and transmitting a remarkable collection of documents and data on rough walls.

\section{Appendix A. impact of $a$}

Notations have been chosen to stay true to Suga et al. ones and ease the transition from one paper to another.

To identify the impact on coefficient $a$, see eq. (29), case $y_{v_{r}}^{*}<y_{0}^{*}$ and $y_{n}>h_{s}^{*}$ is detailed hereinafter. We begin with:

$$
\frac{\partial T}{\partial y^{*}}=\frac{\operatorname{Pr}}{\mu} \frac{C_{T} y^{*}+A_{T}}{1+\Theta_{T}\left(y-y_{v_{r}}^{*}\right)}
$$

and :

$$
\begin{aligned}
& \Theta_{T}=\alpha \frac{\operatorname{Pr}}{P r_{t}}=\alpha \frac{\operatorname{Pr}}{P r_{t_{\infty}}+\mathcal{F}\left(1-\frac{y^{*}}{a h_{s}^{*}}\right)} \text { for } y^{*}<a h_{s} * \\
& \Theta_{T}=\alpha \frac{\operatorname{Pr}}{P r_{t_{\infty}}} \text { for } y^{*} \geq a h_{s}^{*}
\end{aligned}
$$

After integration in the range $\left[y_{0}^{*}, a h_{s}^{*}\right]$ one finds: 


$$
\begin{aligned}
T_{a h}-T_{0} & =-\frac{\operatorname{Pr}}{\mu} \frac{C_{T} \beta_{T} / a}{2\left(\alpha_{T}-\beta_{T} / a\right)}\left(a^{2} h_{s}^{*^{2}}-y_{0}^{*^{2}}\right) \\
& -A_{T} \frac{\operatorname{Pr}}{\mu} \frac{\beta_{T} / a}{\alpha_{T}-\beta_{T} / a}\left(a h_{s}^{*}-y_{0}^{*}\right) \\
& +\frac{\operatorname{Pr} C_{T}}{\mu\left(\alpha_{T}-\beta_{T} / a\right)^{2}} \mathcal{H}^{\alpha \beta}\left(a h_{s}^{*}-y_{0}^{*}\right) \\
& +A_{T} \frac{\operatorname{Pr}}{\mu}\left(\frac{\alpha_{T} H_{s}^{\beta_{T}, a}}{\left(\alpha_{T}-\beta_{T} / a\right)^{2}}\right) \ln \left[\frac{\Lambda_{a h}^{\alpha \beta}}{\Lambda_{0}^{\alpha \beta}}\right] \\
& +-\frac{\operatorname{Pr}}{\mu} \frac{\lambda_{b} C_{T}}{\left(\alpha_{T}-\beta_{T} / a\right)^{3}} \mathcal{H}^{\alpha \beta} \ln \left[\frac{\Lambda_{a h}^{\alpha \beta}}{\Lambda_{0}^{\alpha \beta}}\right]
\end{aligned}
$$

whereas for $y^{*} \in\left[a h_{s}^{*}, y_{n}^{*}\right]$ one gets:

$$
T_{n}-T_{a h}=\frac{\operatorname{Pr}}{\mu} \frac{C_{T}}{\alpha_{T}}\left(y_{n}^{*}-a h_{s}^{*}\right)+\frac{\operatorname{Pr}}{\mu}\left(\frac{A_{T}}{\alpha_{T}}-\frac{C_{T}}{\alpha_{T}^{2}} Y_{w}^{\alpha_{T}}\right) \ln \left[\frac{Y_{n}^{\alpha_{T}}}{Y_{a h}^{\alpha_{T}}}\right]
$$

with:

$$
\left\{\begin{array}{l}
\alpha_{T}=\alpha \frac{P r}{P r_{t_{\infty}}} \\
\beta_{T}=\frac{\mathcal{F}}{h_{s}^{*} P r_{t_{\infty}}} \\
Y^{\alpha_{T}}=1+\alpha_{T}\left(y^{*}-y_{v}^{*}\right) \\
Y^{\beta_{T}, a}=1+\beta_{T}\left(y^{*}-y_{v}^{*} / a\right) \\
\lambda_{b}=Y_{w}^{\alpha_{T}}+\beta_{T} h_{s}^{*} \\
\Lambda^{\alpha \beta}=\lambda_{b}+y^{*}\left(\alpha_{T}-\beta_{T} / a\right) \\
\mathcal{H}^{\alpha \beta}=\left(\alpha_{T} H_{s}^{\beta_{T}, 1}-\left(1+\frac{1}{a}\right) \beta_{T} \lambda_{b}\right)
\end{array}\right.
$$

Combining both expressions it is easy to get coefficients $D_{T}$ and $E_{T}$ such 


$$
\text { that } \begin{aligned}
A_{T}=\left(\frac { \mu } { P r } \left(T_{n}\right.\right. & \left.\left.-T_{0}\right)+C_{T} E_{T}\right) D_{T}: \\
D_{T} & =\frac{1}{\alpha_{T}} \ln \left[\frac{Y_{n}^{\alpha_{T}}}{Y_{a h}^{\alpha_{T}}}\right]-\frac{\beta_{T} / a}{\alpha_{T}-\beta_{T} / a}\left(a h_{s}^{*}-y_{0}^{*}\right) \\
& +\frac{\alpha_{T} H_{s}^{\beta_{T}, a}}{\left(\alpha_{T}-\beta_{T} / a\right)^{2}} \ln \left[\frac{\Lambda_{a h}^{\alpha \beta}}{\Lambda_{y_{0}}^{\alpha \beta}}\right] \\
E_{T} & =\frac{1}{\alpha_{T}}\left(a h_{s}^{*}-y_{n}^{*}\right)+\frac{Y_{w}^{\alpha_{T}}}{\alpha_{T}^{2}} \ln \left[\frac{Y_{n}^{\alpha_{T}}}{Y_{a h}^{\alpha_{T}}}\right] \\
& +\frac{\beta_{T} / a}{2\left(\alpha_{T}-\beta_{T} / a\right)}\left(a^{2} h_{s}^{*^{2}}-y_{0}^{*^{2}}\right) \\
& -\frac{1}{\left(\alpha_{T}-\beta_{T} / a\right)^{2}} \mathcal{H}^{\alpha \beta}\left(a h_{s}^{*}-y_{0}^{*}\right) \\
& +\frac{\lambda_{b}}{\left(\alpha_{T}-\beta_{T} / a\right)^{3}} \mathcal{H}^{\alpha \beta} \ln \left[\frac{\Lambda_{a h}^{\alpha \beta}}{\Lambda_{y_{0}}^{\alpha \beta}}\right]
\end{aligned}
$$

Appendix B. case $y_{n}<h_{s}$

Suga et al [3] only consider cases where $y_{P} \geq h_{s}$. After introducing coefficient $a$, it becomes necessary to account for cases where $y_{n} \leq a h_{s}$. The model then reads: 


$$
\begin{aligned}
A_{u} & =\frac{\alpha \mu u_{N}}{\ln \left(\frac{Y_{N}^{*}}{Y_{w}^{*}}\right)} \\
\overline{P_{k}} & =\frac{\alpha \rho k_{P}}{\mu^{3} y_{n}} \int_{y_{0}^{*}}^{y_{n}^{*}}\left(y^{*}-y_{v_{r}}^{*}\right)\left(\frac{A_{u}}{Y^{*}}\right) d y^{*} \\
D_{T} & =-\frac{\beta_{T} / a}{\alpha_{T}-\beta_{T} / a}\left(y_{n}^{*}-y_{0}^{*}\right)+\frac{\alpha_{T} H_{s}^{\beta_{T}, a}}{\left(\alpha_{T}-\beta_{T} / a\right)^{2}} \ln \left[\frac{\Lambda_{y_{n}}^{\alpha \beta}}{\Lambda_{y_{0}}^{\alpha \beta}}\right] \\
E_{T} & =\frac{\beta_{T} / a}{2\left(\alpha_{T}-\beta_{T} / a\right)}\left(y_{n}^{*^{2}}-y_{0}^{*^{2}}\right) \\
& -\frac{1}{\left(\alpha_{T}-\beta_{T} / a\right)^{2}} \mathcal{H}^{\alpha \beta}\left(y_{n}^{*}-y_{0}^{*}\right) \\
& +\frac{\lambda_{b}}{\left(\alpha_{T}-\beta_{T} / a\right)^{3}} \mathcal{H}^{\alpha \beta} \ln \left[\frac{\Lambda_{y_{n}}^{\alpha \beta}}{\Lambda_{y_{0}}^{\alpha \beta}}\right]
\end{aligned}
$$

[1] B. Aupoix, Roughness Corrections for the $k-\omega$ Shear Stress Transport Model: Status and Proposals, Journal of Fluids Engineering 137 (2015) 021202. doi:10.1115/1.4028122.

[2] B. Aupoix, Improved heat transfer predictions on rough surfaces, International Journal of Heat and Fluid Flows 56 (2015) 160-171. doi:10.1016/j.ijheatfluidflow.2015.07.007.

[3] K. Suga, T. Craft, H. Iacovides, An analytical wall-function for turbulent flows and heat transfer over rough walls, International Journal of Heat and Fluid Flows 27 (5) (2006) 852-866.

[4] J. Nikuradse, Strömungsgesetze in rauhen Rohren, Tech. Rep. 361, VDIForschungsheft (July/August 1933).

[5] J. Nikuradse, Laws of flows in rough pipes, Technical Memorandum 1292, NACA, Washington (April 1937). 
[6] F. Menter, Two-equation eddy-viscosity turbulence models for engineering applications, AIAA Journal 32 (8) (1994) 1598-1605.

[7] P. Villedieu, P. Trontin, R. Chauvin, Glaciated and mixed-phase ice accretion modeling using ONERA 2D icing suite, in: 6th AIAA Atmospheric and Space Environments Conference, AIAA Aviation, Atlanta, GA, 2014.

[8] P. Trontin, A. Kontogiannis, G. Blanchard, P. Villedieu, Description and assessment of the new ONERA 2D icing suite IGLOO2D, in: 9th AIAA Atmospheric and Space Environments Conference, AIAA AVIATION Forum, Denver, Colorado, 2017.

[9] E. Radenac, Validation of a 3D ice accretion tool on swept wings of the SUNSET2 program, in: AIAA AVIATION Forum, 8th AIAA Atmospheric and Space Environments Conference, Washington, D.C., 2016.

[10] C. Bayeux, E. Radenac, P. Villedieu, Theory and validation of a 2D finite-volume integral boundary layer method intended for icing applications, in: 47th AIAA Fluid Dynamics Conference, no. AIAA 2017-3976, AIAA AVIATION Forum, Denver, Colorado, 2017.

[11] N. Bempedelis, C. Bayeux, G. Blanchard, E. Radenac, P. Villedieu, A 3D finite-volume integral boundary layer method for icing applications, in: 9th AIAA Atmospheric and Space Environments Conference, no. AIAA 2017-3417, AIAA AVIATION Forum, Denver, Colorado, 2017.

[12] T. Craft, A. Gerasimov, H. Iacovides, B. Launder, Progress in the gener- 
alization of wall-function treatments, International Journal of Heat and Fluid Flows 23 (2002) 148-160.

[13] A. Perry, W. Schofield, P. Joubert, Rough wall turbulent boundary layers, Journal of Fluid Mechanics 37 (1969) 383.

[14] B. Aupoix, P. Spalart, Extensions of the Spalart-Allmaras turbulence model to account for wall roughness, International Journal of Heat and Fluid Flows 24 (2003) 454-462.

[15] T. Knopp, B. Eisfeld, J. Calvo, A new extension for $k-\omega$ turbulence models to account for wall roughness, International Journal of Heat and Fluid Flow 30 (2009) 54-65.

[16] C. Grigson, Drag losses of new ships caused by hull finish, Journal of Ship Research 36 (2) (1992) 182-196.

[17] C. Colebrook, C. White, Experiments with fluid friction in roughened pipes, Proceedings of the Royal Society of London A 161 (1937) 367-381.

[18] C. Colebrook, Turbulent flow in pipes, with particular reference to the transition region between the smooth and rough pipe laws, Journal of the Institution of Civil Engineering 21 (1938-1939) 133-156.

[19] H. Reichardt, Vollstndige darstellung der turbulenten geschwindigkeitsverteilung in glatten leitungen, Z. Angew. Math. Mech. 31 (1951) 208-219.

[20] A. Refloch, B. Courbet, A. Murrone, P. Villedieu, C. Laurent, 
P. Gilbank, J. Troyes, L. Tessé, G. Chaineray, J. Dargaud, E. Quémerais, F. Vuillot, CEDRE software, Aerospace Lab 2.

[21] F. Chedevergne, Advanced wall model for aerothermodynamics, International Journal of Heat and Fluid Flow 31 (5) (2010) 916-924.

[22] M. Hosni, H. Coleman, R. Taylor, Measurements and calculations of rough-wall heat transfer in the turbulent boundary layer, International Journal of Heat and Mass Transfer 34 (4/5) (1991) 1067-1082.

[23] D. Waigh, R. Kind, Improved aerodynamic characterization of regular three-dimensional roughness, AIAA Journal 36 (6) (1998) 1117-1119.

[24] M. Hosni, H. Coleman, R. Taylor, Measurements and calculation of surface roughness effects on turbulent flow and heat transfer, Tech. Rep. TFD-89-1, Thermal \& Fluid Dynamics Laboratory, Mechanical and Nuclear Engineering Department, Mississippi State University (December 1989).

[25] P. Jackson, On the displacement height in the logarithmic velocity profile, Journal of Fluid Mechanics 111 (1981) 15-25.

[26] N. Dukhan, K. Masiulaniec, K. De Witt, G. van Fossen Jr., Experimental heat transfer coefficients from ice-roughened surfaces for air deicing design, Journal of Aircraft 36 (6) (1999) 948-956.

[27] R. Dirling, Jr., A method for computing rough wall heat transfer rates on reentry nosetips, AIAA Paper 73-763, AIAA $8^{\text {th }}$ Thermophysics Conference, Palm Springs, California (July 16-18 1973). 